\title{
ARMAZENAMENTO REFRIGERADO SOB ATMOSFERA MODIFICADA DE PEDÚNCULOS DE CAJUEIRO-ANÃO-PRECOCE DOS CLONES CCP-76, END-157, END-183 E END-1891
}

\author{
AURICÉLIA DE SOUZA MORAIS ${ }^{1}$, GERALDO ARRAES MAIA ${ }^{2}$, RAIMUNDO WILANE DE FIGUEIREDO ${ }^{3}$, RICARDO \\ ELESBÃO ALVES ${ }^{3}$, HELOÍSA ALMEIDA CUNHA FILGUEIRAS ${ }^{4}$, CARLOS FARLEY HERBSTER MOURA $^{2}$
}

\begin{abstract}
RESUMO - Pedúnculos de clones de cajueiro-anão-precoce, dos clones CCP-76, END-157, END-183 e END-189, foram armazenados durante 25 dias, sob refrigeração associada a atmosfera modificada, com o objetivo de avaliar o efeito das condições atualmente utilizadas $\left(5^{\circ} \mathrm{C}\right.$ e $85-90 \%$ de UR) para conservação, transporte e comercialização de cajus in natura. Os pedúnculos foram avaliados a cada 5 dias quanto às seguintes características: perda de peso, firmeza, cor (antocianinas), sólidos e açúcares solúveis, acidez, $\mathrm{pH}$, vitamina $\mathrm{C}$ e compostos fenólicos. O uso da refrigeração $\left(5^{\circ} \mathrm{C}\right.$ e $85 \%$ U.R), associada a atmosfera modificada, proporcionou uma vida útil pós-colheita de 10 dias para o clone END 189, de até 15 dias para o clone END 157 e de até 25 dias para os clones CCP 76 e END 183. A temperatura de $5^{\circ} \mathrm{C}$ não se mostrou adequada para armazenamento de pedúnculos de coloração mais intensa (END 157 e END 189) que a testemunha (CCP 76), provocando perda de cor (antocianinas) a partir do $10^{\circ}$ dia de armazenamento. A atmosfera modificada reduziu significativamente a perda de peso, favorecendo a aparência do produto para comercialização, independentemente do clone estudado.
\end{abstract}

Termos para indexação: caju, clones, armazenamento, atmosfera modificada.

\section{STORAGE OF CASHEW APPLES FROM DWARF CLONES CCP-76, END-157, END-183 AND END-189 UNDER REFRIGERATION AND MODIFIED ATMOSPHERE}

\begin{abstract}
Apples or false fruits from early dwarf cashew clones CCP-76, END-157, END-183 and END-189 were stored for 25 days under refrigeration associated to modified atmosphere with the aim of evaluating the effect of the conditions presently adopted $\left(5^{\circ} \mathrm{C}\right.$ and $\left.85-90 \% \mathrm{RH}\right)$ for conservation, storage and commercialization of fresh cashew apples. The cashew apples were evaluated every 5 days for the following characteristics: weight loss, pulp firmness, color (anthocyannins), acidity, $\mathrm{pH}$, soluble solids, vitamin $\mathrm{C}$ and phenolic contents. With the use of refrigeration and modified atmosphere it was possible to attain postharvest storage life of 10 days for clone END 189, 15 days for clone END 157 and up to 25 days for clones CCP 76 and END 183. It was found that $5^{\circ} \mathrm{C}$ was not an adequate temperature to store cashew apples with deeper color (END 157 and END 189 ) than the control (CCP 76), as it caused loss of anthocyannins starting from the $10^{\text {th }}$ day of storage. Weight loss was significantly reduced by the use of modified atmosphere, favoring the appearance of the cashew apples for market, independently of the clone.
\end{abstract}

Index terms: Cashew apple, clones, storage, modified atmosphere.

\section{INTRODUÇÃO}

O crescimento do consumo de pedúnculo de cajueiro-anão-precoce (Anacardium occidentale $\mathrm{L}$. var. nanum) como fruta de mesa vem aumentando consideravelmente a cada safra, tanto pela abertura de novos mercados como pela consolidação dos mercados tradicionais (Filgueiras \& Alves, 2001). Isto ocorreu devido principalmente aos novos plantios feitos com cajueiro-anão-precoce que, por apresentarem porte baixo, permitem a colheita manual com maior aproveitamento e redução de perdas (Paiva et al., 1998).

Até muito recentemente, os pedúnculos eram vendidos exclusivamente em feiras locais, porém hoje alcançam supermercados em outras partes do País, localizadas a mais de $4.000 \mathrm{~km}$ do local de produção, podendo ser mantidos em boas condições por até quinze dias após a colheita (Filgueiras et al., 1999), devido ao desenvolvimento de técnicas adequadas de manuseio e conservação pós-colheita, como o uso da refrigeração associada a modificação da atmosfera. Nestas condições, a conservação pós-colheita de pedúnculos de caju, que em temperatura ambiente é de apenas 1 dia, aumentaria para, pelo menos, 10 dias (Menezes, 1992; Menezes \& Alves, 1995; Alves et al., 1998; Figueiredo, 2000).

Paiva et al. (1998) destacaram que, na seleção do clone para a produção de pedúnculos com características comerciais, a qualidade do produto é de fundamental importância. Com a grande variabilidade genética existente, faz-se necessário selecionar pedúnculos que atendam às exigências da comercialização, tais como: alta resistência ao manuseio, avaliada através da firmeza; formato piriforme, de fácil disposição nas embalagens utilizadas; altos teores de açúcar e vitamina C; baixa adstringência; e coloração vermelha (Alves et al., 1998). Além disso, o consumidor prefere pedúnculos de cor laranja a vermelha e de tamanho grande, ou seja, dos tipos 4 ou 5 (de acordo com o número de cajus/ bandeja). Esses tipos alcançam os melhores preços no mercado.

A partir dos genótipos em estudo no Programa de Melhoramento Genético do Cajueiro-Anão-Precoce da Embrapa Agroindústria Tropical, Moura (1998) selecionou, além do CCP 76, tradicionalmente plantado para o mercado de frutos in natura, os clones END 157, END $189 \mathrm{e}$ END 183 como os mais promissores.

O presente trabalho tem como objetivo avaliar o uso das condições de armazenamento atualmente utilizadas a nível comercial, ou seja, refrigeração $\left(5^{\circ} \mathrm{C}\right)$ associada a atmosfera modificada por filme plástico, sobre a conservação pós-colheita de pedúnculos dos clones de cajueiro-anão-precoce CCP 76, END 157, END 183 e END 189.

\section{MATERIALEMÉTODOS}

Os pedúnculos foram obtidos de um experimento de competição de clones de cajueiro-anão-precoce em avaliação no Programa de Melhoramento da Embrapa Agroindústria Tropical, instalado sob irrigação na Empresa Mossoró Agroindustrial S.A. (MAISA), em Mossoró-RN. Foram avaliados quatro clones (CCP 76, END 157, END 183 e END 189), selecionados em experimento anterior realizado por Moura (1998), sendo o CCP-76 utilizado como testemunha, por ser o mais cultivado atualmente para o mercado de mesa.

Os cajus foram colhidos manualmente, no período mais fresco da manhã, e colocados em caixa de colheita em apenas uma camada, para evitar danos físicos. Em seguida, foram transportados até o Laboratório de Fisiologia e Tecnologia de Pós-Colheita da Embrapa Agroindústria Tropical, onde foram submetidos a uma lavagem com hipoclorito de

\footnotetext{
1 (Trabalho 041/2002). Recebido: 09/03/2002. Aceito para publicação: 24/06/2002.

Parte da Dissertação de Mestrado apresentada pelo primeiro autor ao DTA/UFC e financiada com recursos do FUNDECI/BN

2 Bolsista da FUNCAP. UFC.

3 Professor, DTA/UFC, CP 12.168, 60356-000, Fortaleza, CE, frutos @ufc.br

4 Pesquisador, Bolsista PQ-CNPq, Embrapa Agroindústria Tropical, CP 3761, 60511-110, Fortaleza, CE, elesbao@cnpat.embrapa.br
} 
sódio a 50ppm e colocados para secar em temperatura ambiente. Os cajus, em número de quatro, foram dispostos em bandeja de isopor e envolvidos com filme plástico (atmosfera modificada) de PVC (15 micra). Após a pesagem das bandejas, as mesmas foram submetidas a refrigeração $\left(5 \pm 1^{\circ} \mathrm{C}\right.$ e $85 \pm 3 \%$ de U.R.) em câmaras que eram diariamente monitoradas através de um termoigrômetro. No tempo 0 (colheita), e a cada cinco dias, três bandejas eram retiradas, pesadas e os pedúnculos avaliados quanto à aparência (senescência) e firmeza. Em seguida, os mesmos foram cortados em quatro pedaços iguais no sentido longitudinal e distribuídos em sacos transparentes de polietileno de baixa densidade com fecho hermético, devidamente identificados e armazenados em "freezer" $\left(-20^{\circ} \mathrm{C}\right)$ para posteriores análises físico-químicas e químicas.

As avaliações realizadas foram as seguintes: perda de peso - em balança semi-analítica; firmeza da polpa - com penetrômetro manual FT011; senescência - escala de notas de 0-4 ( 0 = pedúnculos com aparência boa, resistente ao choque mecânico, isento de cheiro e enrugamento; 1 = manchas pequenas de mudança de cor $; 2=$ descoloração em aproximadamente metade do pedúnculo; 3 = perda de brilho, mudança total de coloração e presença de depressão, característica de contato com a embalagem; e 4 = liberação espontânea do suco, presença de fungos, cheiro desagradável, imprestável para o consumo), sugerida por Menezes (1992) e Figueiredo (2000); sólidos solúveis totais (SST), acidez total titulável (ATT) e pH (IAL, 1985); açúcares solúveis totais (AST), conforme Yemn \& Willis (1954); vitamina C total (Strohecker \& Henning, 1967); compostos fenólicos (Reicher et al., 1981); e antocianinas totais (Francis, 1982).

$\mathrm{O}$ experimento foi conduzido em delineamento inteiramente casualizado (DIC), em esquema fatorial 4X6 (clones x tempo de armazenamento). Foram utilizadas 3 repetições, representadas por cada unidade experimental, ou seja, bandeja de isopor contendo 4 cajus. A partir dos resultados preliminares da análise de variância, verificando-se interação significativa entre os fatores, o tempo foi desdobrado dentro de cada clone e os resultados submetidos à regressão polinomial, considerando-se equações de até $3^{\circ}$ grau. O coeficiente de determinação mínimo para a utilização das curvas foi de 0,70 .

\section{RESULTADOSE DISCUSSÃO}

De forma geral, as análises de variância revelaram interações significativas para as características avaliadas, com exceção de SST, perda de peso e firmeza da polpa, para as quais houve efeito significativo do tempo e, para firmeza, que também houve efeito dos clones.

O uso da atmosfera modificada, independentemente dos clones, propiciou uma perda de, peso de no máximo, $1,4 \%$ ao final do período de armazenamento (Figura 1A), podendo ser considerada baixa para o caju, pois não resulta em nenhum sintoma de murchamento. Resultados semelhantes foram encontrados por Menezes (1992), trabalhando com pedúnculos de cajueiro comum, e Figueiredo (2000), em trabalho realizado com pedúnculos de cajueiro-anão-precoce CCP 76, que encontraram, respectivamente, $0,8 \%$ (14 dias) e 1,2\% (25 dias) de perda.

Nos pedúnculos dos clones estudados neste trabalho, houve um decréscimo gradual da firmeza até 15 dias de armazenamento. Após esse tempo, verificou-se que essa característica tendeu à estabilização (Figura 1B). Os clones CCP 76, END 183 e END 189, pelo teste de Tukey a 5\%, não apresentaram diferença estatística; apenas o clone END 157 apresentou pedúnculos mais firmes (dados não apresentados). De modo geral, a firmeza da polpa dos pedúnculos aqui avaliados foi superior à encontrada por Menezes (1992), usando condições de armazenamento semelhantes a esse trabalho.

Em relação à qualidade interna, apesar de ter sido observada interação significativa para a maioria das características (AST, ATT, SST/ ATT e pH), praticamente não houve variação em relação ao tempo e/ou aos clones (dados não apresentados). Os pedúnculos apresentaram, em média: $\mathrm{SST}=10,7^{\circ} \mathrm{Brix} ; \mathrm{AST}=8,6 \%$; ATT $=0,35 \mathrm{~g}$ ácido málico $/ 100 \mathrm{~g}$; $\mathrm{SST} / \mathrm{ATT}=31,25 ; \mathrm{e} \mathrm{pH}=4,6$, valores esses dentro das faixas reportadas por Pinto et al. (1997) e Moura et al. (2000) para pedúnculos de clones de cajueiro-anão-precoce.

Pela escala de senescência utilizada, os pedúnculos que apresentaram nota superior a 2 , já se apresentam com um padrão de qualidade externa inferior (perda de brilho e perda da cor característica), ao exigido pelo mercado; pode-se constatar que os clones END 157 e END 189 (Figura 2A) foram os que mostraram menor vida útil pós-colheita (entre 10 e 15 dias) nas atuais condições comerciais utilizadas, ou seja, $5^{\circ} \mathrm{Ce} 85-90 \%$ de UR.

A essa temperatura, a vida útil dos pedúnculos parece estar associada de forma inversa à intensidade de cor vermelha (Figura 2B). De acordo com Moura et al. (2001), os clones estudados nesse experimento, em termos de intensidade de coloração vermelha, medida através do teor de antocianinas totais na película, seguem a seguinte ordem decrescente: END-189, END-157, CCP-76 e END-183, com teores médios de 76; 59; 37 e $18 \mathrm{mg} / 100 \mathrm{~g}$, respectivamente. Apesar de terem sido observadas nessa pesquisa diferenças quanto aos teores, também foi observada a mesma seqüência. Os resultados obtidos (Figuras 2A e B) indicam que pedúnculos mais vermelhos são mais suscetíveis a danos pelo frio e que, nessas condições, a temperatura utilizada não seria adequada para a conservação dos mesmos, tendo em vista a perda da coloração vermelha característica, avaliada através do conteúdo de antocianinas totais.

Como muitas substâncias podem coexistir com as antocianinas nos vacúolos, não há dúvida de que alguns podem interagir com as mesmas, resultando na prevenção da perda de cor ou igualmente no aumento da cor. A natureza química, concentração, presença de pigmentos, $\mathrm{pH}$, presença de compostos conhecidos como copigmentos e, algumas vezes, a presença de certos íons metálicos, parecem ser os principais fatores que influenciam a cor das antocianinas contidas no meio (Brouillard, 1983; Stafford, 1990).

$\mathrm{O} \mathrm{pH}$, no entanto, é o mais importante fator que afeta a cor das antocianinas (Mazza \& Brouillard, 1987). Agindo como indicadores de $\mathrm{pH}$, as antocianinas são vermelhas com $\mathrm{pH}$ baixo, incolores em $\mathrm{pH}$ intermediário e azuladas em pH elevado (Bate-Smith, 1954; Wrolstad et al. 1970; Mazza \& Brouillard, 1987).

De acordo com Murata (1990), em geral, o principal efeito do armazenamento de frutos sob condições que provocam danos pelos frio, é a separação de fase (transição) dos lipídios polares das membranas celulares e/ou diminuição do transporte ativo para dentro da célula. O repentino aumento na permeabilidade da membrana, no começo do dano pelo frio, pode resultar em desnaturação de membranas, tais como plasmalema e tonoplasto (vacúolo). Em conseqüência disso, o pH mais baixo do conteúdo do vacúolo, em contato com o pH externo, pode sofrer aumento, provocando as alterações observadas (perda de cor).

Segundo Menezes (1992), o uso de ambiente refrigerado para o armazenamento de pedúnculos de caju é decisivo para o prolongamento de sua vida útil pós-colheita, já que, em temperatura ambiente, a vida útil do material é de apenas 1 dia, e em ambiente refrigerado, este período aumentaria para 10 dias.

Pelos resultados obtidos neste experimento, não se observou infecção fúngica durante os 25 dias de armazenamento. De acordo também com Menezes (1992), isso provavelmente ocorre devido à presença de elevados teores de compostos fenólicos, especialmente dímeros e oligoméricos no início do armazenamento (Figura 3A e B).

Houve uma tendência geral de diminuição no teor de todos os compostos fenólicos durante o período de armazenamento (Figura 3A, B e C). Segundo Menezes e Alves (1995), isso geralmente ocorre durante o armazenamento refrigerado de pedúnculos do caju, o que também foi observado por Figueiredo (2000) com pedúnculos do clone CCP-76.

Como para outras características de qualidade interna, a variação no teor de vitamina $\mathrm{C}$ total foi pequena, com exceção do clone END 189 (Figura 3D). A vitamina C está presente nos tecidos vegetais, principalmente na forma de ácido ascórbico reduzido (AA). Esse composto pode ser oxidado, de modo reversível, a ácido deidroascórbico (DHA). De acordo com Mapson (1970), essas oxidações ocorrem via enzimas ou na presença de metais. 


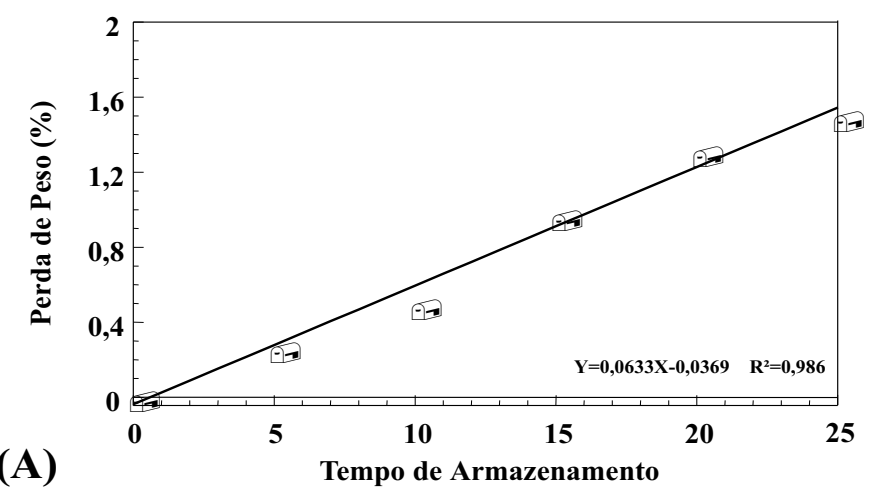

FIGURA 1 - Perda de peso (A) e firmeza (B) de pedúnculos de cajueiro-anão-precoce armazenados sob refrigeração $\left(5^{\circ} \mathrm{C} \pm 1^{\circ} \mathrm{C}\right.$ e umidade relativa de $85 \% \pm 3 \%)$, associada a atmosfera modificada.

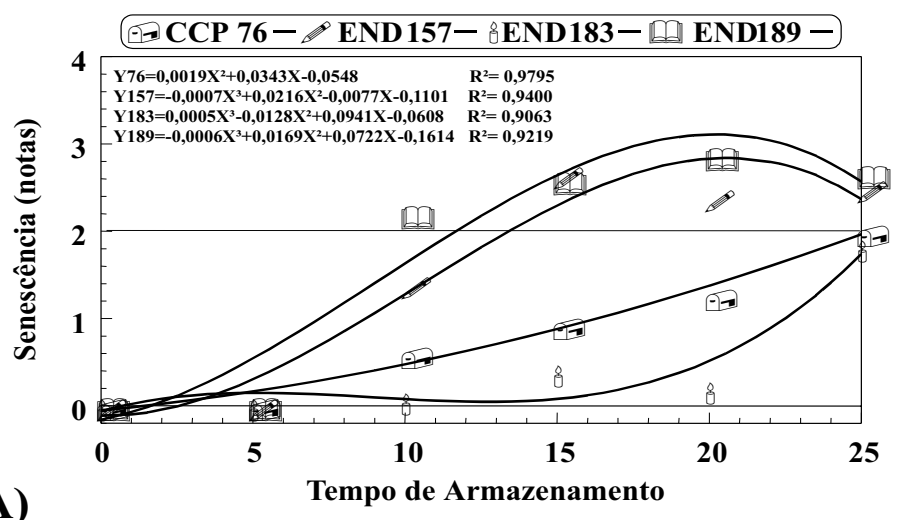

(A)

Tempo de Armazenamento

FIGURA 2 - Senescência (A) e antocianinas (B) de pedúnculos de quatro clones de cajueiro-anão-precoce armazenados sob refrigeração $\left(5^{\circ} \mathrm{C} \pm 1^{\circ} \mathrm{C}\right.$ e umidade relativa de $85 \% \pm 3 \%$ ), associada a atmosfera modificada.

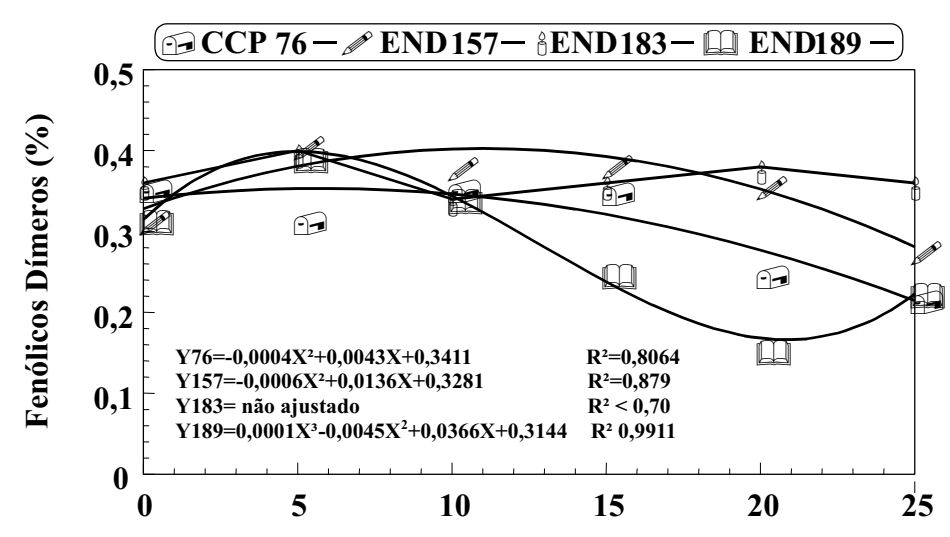

(A)

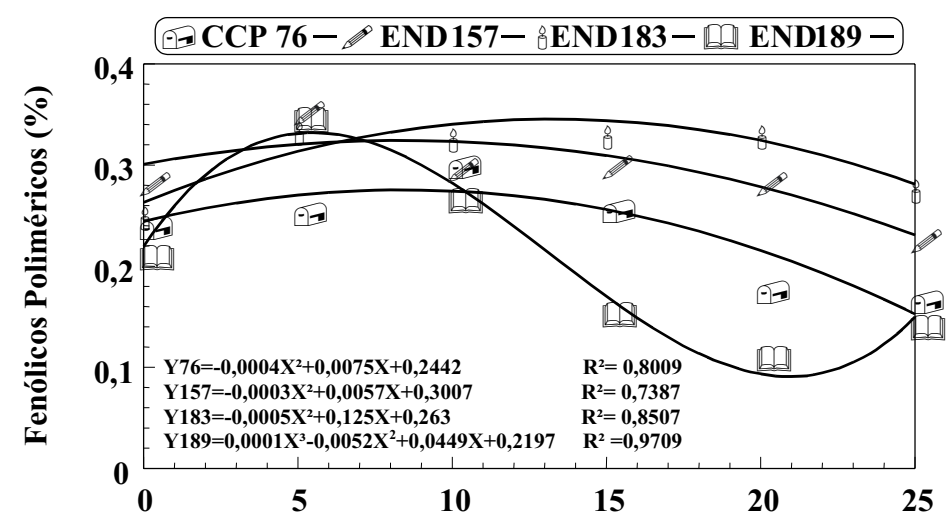

(C) Tempo de Armazenamento (dias)

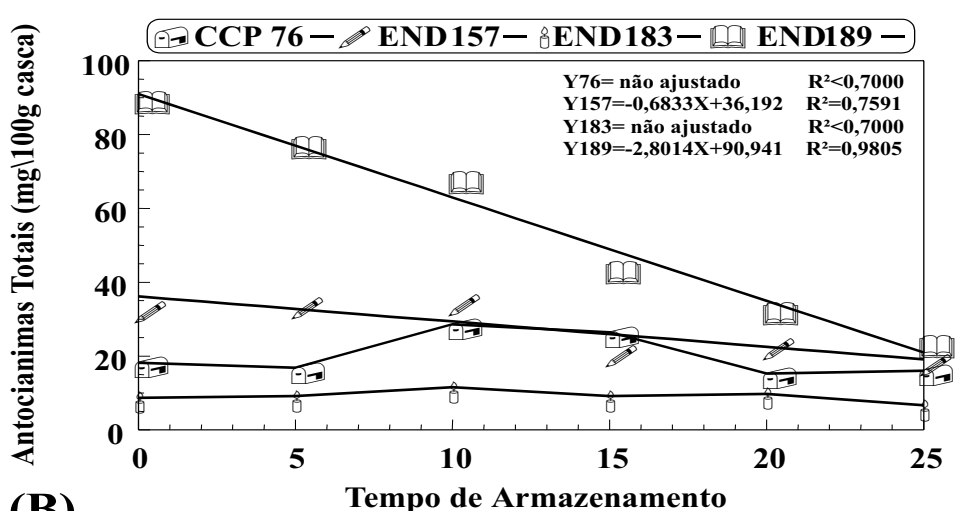

(B)

Tempo de Armazenamento

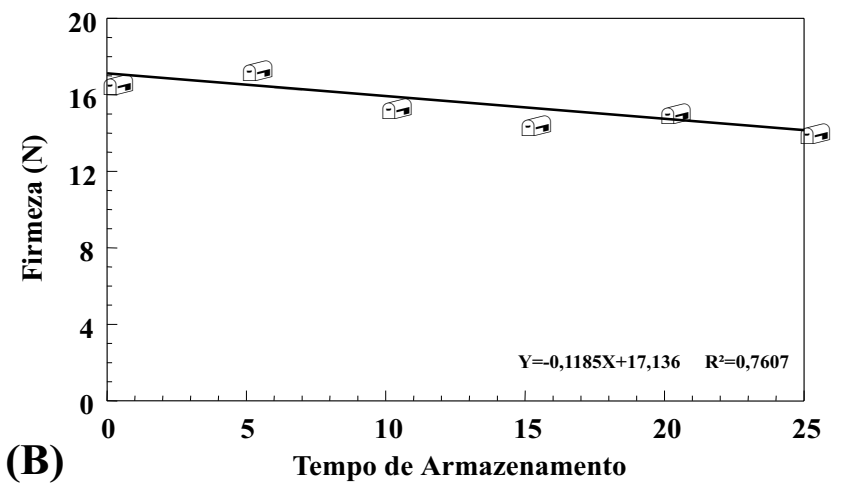

(B)
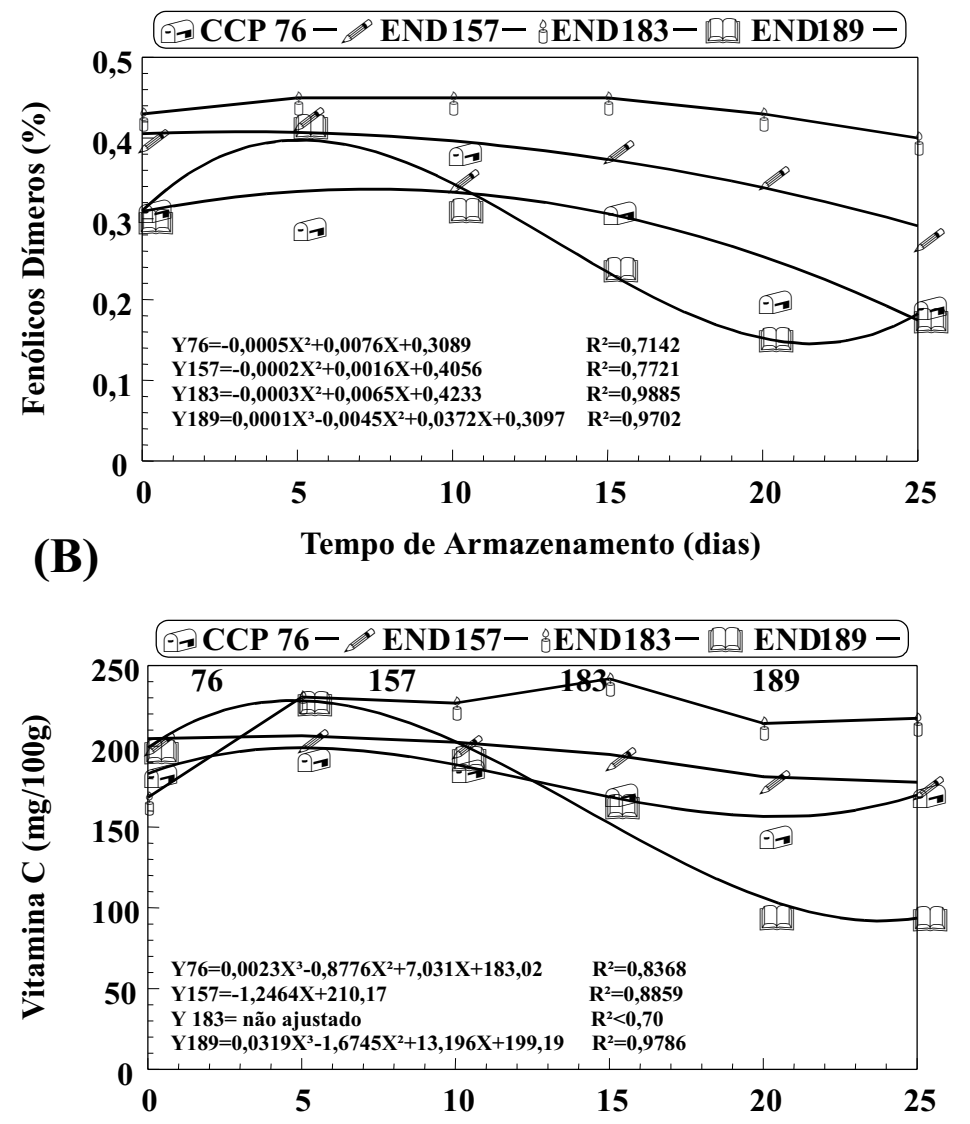

(D)

Tempo de Armazenamento (dias)

FIGURA 3 - Compostos fenólicos dímeros (A), oligoméricos (B) e poliméricos (C) e Vitamina C (D) de pedúnculos de quatro clones de cajueiro-anãoprecoce armazenados sob refrigeração $\left(5^{\circ} \mathrm{C} \pm 1^{\circ} \mathrm{C}\right.$ e umidade relativa de $\left.85 \% \pm 3 \%\right)$, associada a atmosfera modificada. 
Geralmente, a modificação da atmosfera resulta numa maior retenção de ácido ascórbico, devido principalmente à menor disponibilidade de $\mathrm{O}_{2}$ no interior da embalagem (Kader, 1986), o que não aconteceu no END, 189 que sofreu mais danos pelo frio. Esse clone, assim como para vitamina $\mathrm{C}$, também apresentou uma redução mais pronunciada nos compostos fenólicos ao final do experimento.

\section{CONCLUSÕES}

1. O uso da refrigeração $\left(5^{\circ} \mathrm{C}\right.$ e $85 \%$ U.R $)$, associada a atmosfera modificada, proporcionou uma vida útil pós-colheita de até 10 dias para o clone END 189, 15 dias para o clone END 157 e de até 25 dias para os clones CCP 76 e END 183.

2. A temperatura de $5^{\circ} \mathrm{C}$ não se mostrou adequada para armazenamento de pedúnculos de coloração mais intensa (END 157 e END 189) que a testemunha (CCP 76), provocando perda de cor (antocianinas) a partir do $10^{\circ}$ dia de armazenamento.

\section{REFERÊNCIASBIBLIOGRÁFICAS}

ALVES, R.E.; FILGUEIRAS, H.A.C.; MOSCA, J.L.; M., J.B.; LIMA, I.M.L.S.; ALMEIDA, J.H.S.; SOUZA FILHO, M.S.M.; LIMA, A.C.; MELO, Q.M.S.; FREIRE, F.C.O.. Colheita e conservação pós-colheita do pedúnculo e da castanha de caju. In: SILVA, V.V. 500 Perguntas e 500 Respostas - Caju. Fortaleza: Embrapa Agroindústria Tropical, 1998. p.135-162.

BATE-SMITH, E.C. Flavonoid compounds in foods. Advances in Food Research, New York, v.5, p.261-300, 1954.

BROUILLARD, R. The in vivo expression of anthocyanin colour in plants. Phytochemistry, Elmsford, v.22, n.6, p.1311-1323, 1983.

FIGUEIREDO, R.W. Qualidade e bioquímica de parede celular durante o desenvolvimento, maturação e armazenamento de pedúnculos de cajueiro-anão-precoce CCP-76 submetidos à aplicação pós-colheita de cálcio. 2000. 154f. (Doutorado em Ciência dos Alimentos) - Faculdade de Ciências Farmacêuticas, Universidade de São Paulo, São Paulo, 2000.

FILGUEIRAS, H.A.C.; ALVES, R.E. Cashew apple for fresh consumption in Brazil. In: WORLD CASHEW CONGRESS, 1., 2001, Kochi (Índia). Cashew - The millennium nut: Kochi: CEPC, 2001. p. 53-55.

FILGUEIRAS, H.A.C.; ALVES, R.E.; MOSCA, J.L.; MENEZES, J.B. Cashew apple for fresh consumption: research on harvest and postharvest handling technology in Brazil. Acta Horticulturae, Leuven, n.485, p.155-160, 1999.

FRANCIS, F.J. Analysis of anthocyanins. In: MARKAKIS, P. Anthocyanins as food colors. New York: Academic, 1982. p.181-207.

INSTITUTO ADOLFO LUTZ. Normas analíticas, métodos químicos e físicos para análise de alimentos. São Paulo: IAL, 1985. v.1, 533p.

KADER, A.A. Biochemical and physiological basis of effects of controlled and modified atmospheres on fruit and vegetables. Food Technology, Chicago, v.40, n.5, p.99-104, 1986.

MAPSON, L.W. Vitamins in fruits. In: HULME, A . C. The biochemistry of fruits and their products. London: Academic Press, 1970. v.1, cap.13, p.369-382.
MAZZA, G.; BROUILLARD, R. Recent developments in the stabilization of anthocyanins in food products. Food Chemistry, Essex, v.25, n.3, p.207-225, 1987.

MENEZES, J.B. Armazenamento refrigerado de pedúnculos do caju (Anacardium occidentale $\mathbf{L}$.) sob atmosfera ambiental e modificada. 1992. 102f. Dissertação (Mestrado em Ciência dos Alimentos) Escola Superior de Agricultura de Lavras, Lavras, 1992

MENEZES, J.B.; ALVES, R.E. Fisiologia e tecnologia pós-colheita do pedúnculo do caju. Fortaleza: Embrapa Agroindústria Tropical, 1995. 20p. (Documentos, 17).

MOURA, C.F.H. Qualidade de pedúnculos de clones de cajueiro-anãoprecoce (Anarcadium occidentale L. var. nanum) irrigados. 1998. 96f. Dissertação (Mestrado em Fitotecnia) - Universidade Federal do Ceará, Fortaleza, 1998.

MOURA, C.F.H.; ALVES, R.E.; FILGUEIRAS, H.A.C.; INEECCO, R.; PINTO, S.A.A. Internal quality of new dwarf cashew apple (Anacardium occidentale L. var. nanum) clones cultivated under irrigation and destined to fresh consumption. Proceedings of the Interamerican Society for Tropical Horticulture, Homestead, v.42, p.119-123, 2000.

MOURA, C.F.H.; ALVES, R.E.; INEECCO, R.; FILGUEIRAS, H.A.C.; MOSCA, J.L.; PINTO, S.A.A. Características físicas de pedúnculos de cajueiro-anão para comercialização in natura. Revista Brasileira de Fruticultura, Jaboticabal, SP, v. 23, n.3, p. 537-540, 2001.

MURATA, T. Relation of chilling stress to membrane permeability. In: WANG, C.Y. Chilling injury of horticultural crops. Boca Raton: CRC, 1990. p.201-209.

PAIVA, J. R.; ALVES, R. E.; BARROS, L.M.; CAVALCANTI, J.J.V.; ALMEIDA, J.H.S.; MOURA, C. F. H. Produção e qualidade de pedúnculos de clones de cajueiro-anão-precoce sob cultivo irrigado. Fortaleza: Embrapa Agroindústria Tropical, 1998. 5p. (Comunicado Técnico, 19).

PINTO, S.A.A.; ALVES, R.E.; MOSCA, J.L.; FILGUEIRAS, H.A.C.; MOURA, C.F.H. Fresh consumption quality of the apple of some Brazilian early dwarf cashew clones (Anacardium occidentale). Proceedings of the Interamerican Society For Tropical Horticulture, Homestead, v.41, p.189-193, 1997.

REICHER, F.; SIERAKOWSKI, M.R.; CORREA, J.B.C. Determinação espectrofotométrica de taninos pelo reativo fosfotúngsticofosfomolíbdico. Arquivos de Biologia e Tecnologia, Curitiba, v.24, n.4, p.407-411, 1981.

STAFFORD, H.A. Flavonoid metabolism. Boca Raton: CRC, 1990. 286p. STROHECKER, R.; HENNING, H.M. Análisis de vitaminas: métodos comprobados. Madrid: Paz Montalvo, 1967. 428p.

WROLSTAD, R.E.; PUTNAM, T.P.; VARSEVOLG, G.W. Color quality of frozen strawberries: effect of anthocyanin, $\mathrm{pH}$, total acidity and acid ascorbic. Journal of Food Science, Chicago, v.5, p.448-451, 1970.

YEMN, E.W.; WILLIS, A.J. The estimation of carbohydrate in plant extracts by antrone. The Biochemical Journal, London, v.57, p.508$514,1954$. 